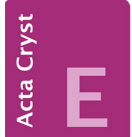
COMMUNICATIONS

ISSN 2056-9890

\section{Crystal structure of pseudoguainolide}

\author{
Noureddine Beghidja, ${ }^{a} *$ Samir Benayache, ${ }^{a}$ Fadila \\ Benayache, ${ }^{a}$ David W. Knight ${ }^{b}$ and Benson M. Kariuki ${ }^{b *}$ \\ a Unité de Recherche VARENBIOMOL, Constantine 1 University, Constantine 25000, \\ Algeria, and ${ }^{\mathbf{b}}$ School of Chemistry, Cardiff University, Main Building, Park Place, \\ Cardiff CF10 3AT, Wales. *Correspondence e-mail: nourbeghidja@yahoo.fr, \\ kariukib@cf.ac.uk
}

Received 8 January 2015; accepted 5 February 2015

Edited by E. R. T. Tiekink, University of Malaya, Malaysia

The lactone ring in the title molecule, $\mathrm{C}_{15} \mathrm{H}_{22} \mathrm{O}_{3}$ (systematic name: 3,4a,8-trimethyldodecahydroazuleno[6,5- $b$ ]furan-2,5dione), assumes an envelope conformation with the methine $\mathrm{C}$ atom adjacent to the the methine $\mathrm{C}$ atom carrying the methyl substituent being the flap atom. The other fivemembered ring adopts a twisted conformation with the twist being about the methine-methylene $\mathrm{C}-\mathrm{C}$ bond. The sevenmembered ring is based on a twisted boat conformation. No specific interactions are noted in the the crystal packing.

Keywords: crystal structure; plant extract; inula graveolens.

CCDC reference: 1047797

\section{Related literature}

For background to inula graveolens, see: Chiappini \& Fardella (1980); Rustaiyan et al. (1987). For related structures, see: Herz et al. (1982); Schmidt et al. (1996); Wu et al. (2012); Billodeaux et al. (2014).

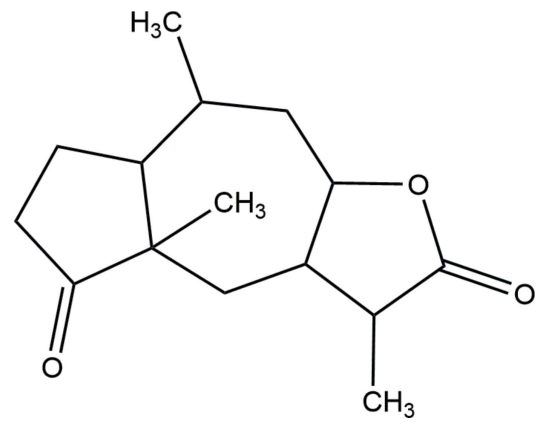

\section{Experimental}

2.1. Crystal data

$\mathrm{C}_{15} \mathrm{H}_{22} \mathrm{O}_{3}$

$M_{r}=250.33$

Orthorhombic, $P 2_{1} 2_{1} 2_{1}$

$a=7.4320(3) \AA$

$b=11.9278(3) \AA$

$c=15.3152(6) \AA$

$V=1357.65(8) \AA^{3}$

$Z=4$

Mo $K \alpha$ radiation

$\mu=0.08 \mathrm{~mm}^{-1}$

$T=150 \mathrm{~K}$

$0.20 \times 0.20 \times 0.04 \mathrm{~mm}$

\subsection{Data collection}

Nonius KappaCCD diffractometer Absorption correction: multi-scan (DENZO/SCALEPACK; Otwinowski \& Minor, 1997)

$T_{\min }=0.984, T_{\max }=0.997$

9382 measured reflections 3098 independent reflections 2533 reflections with $I>2 \sigma(I)$ $R_{\text {int }}=0.041$

\subsection{Refinement}

$R\left[F^{2}>2 \sigma\left(F^{2}\right)\right]=0.047$

$w R\left(F^{2}\right)=0.107$

$S=1.08$

3098 reflections

166 parameters

$\mathrm{H}$-atom parameters constrained

$\Delta \rho_{\max }=0.15 \mathrm{e} \AA^{-3}$

$\Delta \rho_{\min }=-0.16$ e $\AA^{-3}$

Data collection: COLLECT (Nonius, 2000); cell refinement: SCALEPACK (Otwinowski \& Minor, 1997); data reduction: DENZO (Otwinowski \& Minor, 1997) and SCALEPACK; program(s) used to solve structure: SIR92 (Altomare et al., 1999); program(s) used to refine structure: SHELXL97 (Sheldrick, 2008); molecular graphics: ORTEP-3 for Windows (Farrugia, 2012); software used to prepare material for publication: WinGX (Farrugia, 2012).

\section{Acknowledgements}

The authors extend their appreciation to Constantine 1 University and Cardiff University for supporting this research.

Supporting information for this paper is available from the IUCr electronic archives (Reference: TK5358).

\section{References}

Altomare, A., Burla, M. C., Camalli, M., Cascarano, G. L., Giacovazzo, C., Guagliardi, A., Moliterni, A. G. G., Polidori, G. \& Spagna, R. (1999). J. Appl. Cryst. 32, 115-119.

Billodeaux, D. R., Menelaou, M. A., Fischer, N. H. \& Fronczek, F. R. (2014). Private communication (deposition number CCDC 989352). CCDC, Cambridge, England.

Chiappini, I. \& Fardella, G. (1980). Fitoterapia, 51, 161-162.

Farrugia, L. J. (2012). J. Appl. Cryst. 45, 849-854.

Herz, W., Watanabe, K. \& Blount, J. F. (1982). J. Org. Chem. 47, 3011-3012.

Nonius (2000). COLLECT. Nonius BV, Delft, The Netherlands.

Otwinowski, Z. \& Minor, W. (1997). Methods in Enzymology, Vol. 276, Macromolecular Crystallography, Part A, edited by C. W. Carter Jr \& R. M. Sweet, pp. 307-326. New York: Academic Press.

Rustaiyan, A., Jakupovic, J., Chau-Thi, T. V., Bohlmann, F. \& Sadjadi, A. (1987). Phytochemistry, 26, 2603-2606.

Schmidt, T. J., Fronczek, F. R. \& Liu, Y.-H. (1996). J. Mol. Struct. 385, 113-121.

Sheldrick, G. M. (2008). Acta Cryst. A64, 112-122.

Wu, P., Su, M.-X., Wang, Y., Wang, G.-C., Ye, W.-C., Chung, H.-Y., Li, J., Jiang, R.-W. \& Li, Y.-L. (2012). Phytochemistry, 76, 133-140. 


\section{supporting information}

Acta Cryst. (2015). E71, o162 [doi:10.1107/S2056989015002510]

\section{Crystal structure of pseudoguainolide}

\section{Noureddine Beghidja, Samir Benayache, Fadila Benayache, David W. Knight and Benson M. Kariuki}

\section{S1. Comment}

Inula graveolens have consistently been the subject of research interest (Chiappini \& Fardella, 1980; Rustaiyan et al., 1987). Our interest is in the extracts from aerial parts of Algerian species such as stems, flowers and leaves. The asymmetric unit of the crystal structure consists of a single molecule (Fig. 1). In the molecule, the lactone ring assumes an envelope conformation. In the crystal structure, the planes of the lactone rings are approximately parallel. The molecules are arranged with the lactone rings stacked parallel to the $a$ axis. Structures of some related compounds have been reported (Herz et al., 1982; Schmidt et al., 1996; Wu et al., 2012; Billodeaux et al., 2014).

\section{S2. Experimental}

The air-dried aerial parts of inula graveolens $(500 \mathrm{~g})$ were extracted with acetone/ $\mathrm{Et}_{2} \mathrm{O}(1: 1)$ at room temperature. The solution was filtered off and concentrated under reduced pressure to give a pale-yellow gum $(9 \mathrm{~g})$. The gum was subjected to successive column chromatography (silica gel) and TLC (silica gel, PF254). Eleven fractions were obtained. Fraction 5 gave a material which crystallized as colourless crystals with a melting point of $152{ }^{\circ} \mathrm{C}$.

\section{S3. Refinement}

Carbon-bound $\mathrm{H}$-atoms were placed in calculated positions $(\mathrm{C}-\mathrm{H}=0.96-0.98 \AA)$ and were included in the refinement in the riding model approximation, with $U_{\text {iso }}(\mathrm{H})$ set to $1.2-1.5 U_{\text {equiv }}(\mathrm{C})$. 


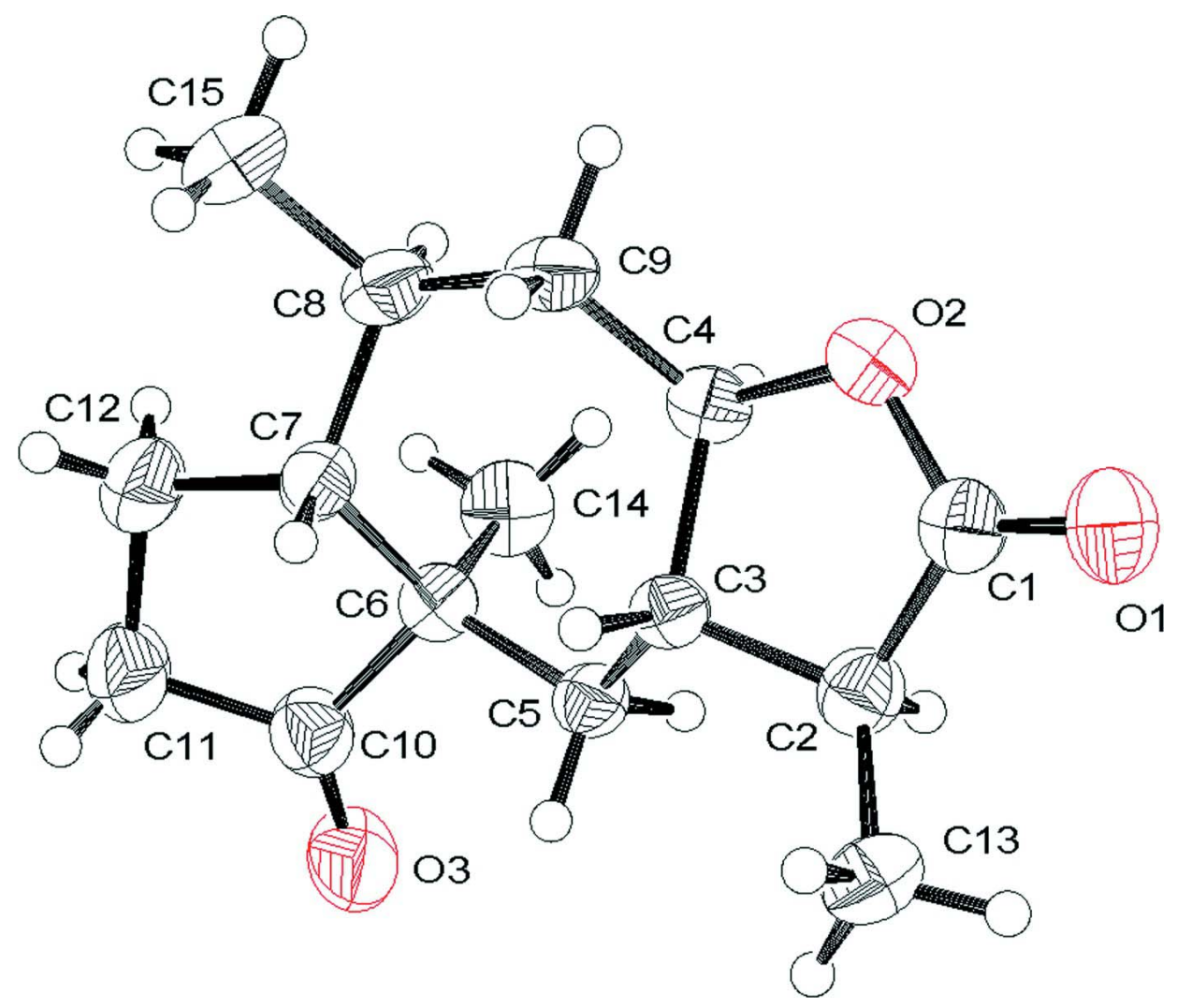

\section{Figure 1}

A molecule showing atom labels and 50\% probability displacement ellipsoids for non-H atoms.

\section{3,4a,8-Trimethyldodecahydroazuleno[6,5-b] furan-2,5-dione}

Crystal data

$\mathrm{C}_{15} \mathrm{H}_{22} \mathrm{O}_{3}$

$M_{r}=250.33$

Orthorhombic, $P 2{ }_{1} 2_{1} 2_{1}$

$a=7.4320(3) \AA$

$b=11.9278(3) \AA$

$c=15.3152(6) \AA$

$V=1357.65(8) \AA^{3}$

$Z=4$

$F(000)=544$

Data collection

\section{Nonius KappaCCD}

diffractometer

Radiation source: fine-focus sealed tube

Graphite monochromator

CCD slices, $\omega$ and $\varphi$ scans

Absorption correction: multi-scan

(DENZO/SCALEPACK; Otwinowski \& Minor, 1997)

$T_{\min }=0.984, T_{\max }=0.997$
$D_{\mathrm{x}}=1.225 \mathrm{Mg} \mathrm{m}^{-3}$

Mo $K \alpha$ radiation, $\lambda=0.71073 \AA$

Cell parameters from 3098 reflections

$\theta=3.1-27.5^{\circ}$

$\mu=0.08 \mathrm{~mm}^{-1}$

$T=150 \mathrm{~K}$

Plate, colourless

$0.20 \times 0.20 \times 0.04 \mathrm{~mm}$

9382 measured reflections

3098 independent reflections

2533 reflections with $I>2 \sigma(I)$

$R_{\text {int }}=0.041$

$\theta_{\text {max }}=27.5^{\circ}, \theta_{\text {min }}=3.1^{\circ}$

$h=-9 \rightarrow 9$

$k=-15 \rightarrow 15$

$l=-19 \rightarrow 17$ 


\section{Refinement}

Refinement on $F^{2}$

Least-squares matrix: full

$R\left[F^{2}>2 \sigma\left(F^{2}\right)\right]=0.047$

$w R\left(F^{2}\right)=0.107$

$S=1.08$

3098 reflections

166 parameters

0 restraints

Primary atom site location: structure-invariant direct methods
Secondary atom site location: difference Fourier map

Hydrogen site location: inferred from neighbouring sites

$\mathrm{H}$-atom parameters constrained

$w=1 /\left[\sigma^{2}\left(F_{\mathrm{o}}^{2}\right)+(0.0453 P)^{2}+0.2031 P\right]$ where $P=\left(F_{\mathrm{o}}{ }^{2}+2 F_{\mathrm{c}}{ }^{2}\right) / 3$

$(\Delta / \sigma)_{\max }<0.001$

$\Delta \rho_{\max }=0.15 \mathrm{e} \AA^{-3}$

$\Delta \rho_{\min }=-0.16$ e $\AA^{-3}$

Special details

Geometry. All s.u.'s (except the s.u. in the dihedral angle between two l.s. planes) are estimated using the full covariance matrix. The cell s.u.'s are taken into account individually in the estimation of s.u.'s in distances, angles and torsion angles; correlations between s.u.'s in cell parameters are only used when they are defined by crystal symmetry. An approximate (isotropic) treatment of cell s.u.'s is used for estimating s.u.'s involving l.s. planes.

Refinement. Refinement of $F^{2}$ against ALL reflections. The weighted $R$-factor $w R$ and goodness of fit $S$ are based on $F^{2}$, conventional $R$-factors $R$ are based on $F$, with $F$ set to zero for negative $F^{2}$. The threshold expression of $F^{2}>2 \sigma\left(F^{2}\right)$ is used only for calculating $R$-factors (gt) etc. and is not relevant to the choice of reflections for refinement. $R$-factors based on $F^{2}$ are statistically about twice as large as those based on $F$, and $R$ - factors based on ALL data will be even larger.

Fractional atomic coordinates and isotropic or equivalent isotropic displacement parameters $\left(\AA^{2}\right)$

\begin{tabular}{|c|c|c|c|c|}
\hline & $x$ & $y$ & $z$ & $U_{\text {iso }} * / U_{\text {eq }}$ \\
\hline $\mathrm{C} 1$ & $0.8136(2)$ & $0.24699(16)$ & $0.52812(12)$ & $0.0383(4)$ \\
\hline $\mathrm{C} 2$ & 0.8423 & $0.16380(15)$ & $0.45546(12)$ & 0.0407 (4) \\
\hline $\mathrm{H} 2$ & 0.9722 & 0.1503 & 0.4522 & $0.049 *$ \\
\hline $\mathrm{C} 3$ & $0.7918(2)$ & $0.22980(13)$ & $0.37360(11)$ & $0.0318(4)$ \\
\hline H3 & 0.6620 & 0.2220 & 0.3642 & $0.038^{*}$ \\
\hline $\mathrm{C} 4$ & $0.8307(2)$ & $0.35109(14)$ & $0.40005(11)$ & $0.0338(4)$ \\
\hline $\mathrm{H} 4$ & 0.9552 & 0.3703 & 0.3852 & $0.041 *$ \\
\hline $\mathrm{C} 5$ & $0.8884(2)$ & $0.19024(14)$ & $0.29170(12)$ & $0.0346(4)$ \\
\hline H5A & 0.8386 & 0.1183 & 0.2748 & $0.041 *$ \\
\hline H5B & 1.0142 & 0.1785 & 0.3060 & $0.041^{*}$ \\
\hline C6 & $0.8781(2)$ & $0.26974(15)$ & $0.21218(12)$ & $0.0371(4)$ \\
\hline $\mathrm{C} 7$ & $0.7099(3)$ & $0.34762(15)$ & $0.20872(12)$ & $0.0388(4)$ \\
\hline $\mathrm{H} 7$ & 0.6065 & 0.3039 & 0.2292 & $0.047^{*}$ \\
\hline $\mathrm{C} 8$ & $0.7176(3)$ & $0.45618(14)$ & $0.26350(13)$ & $0.0429(5)$ \\
\hline H8 & 0.8326 & 0.4932 & 0.2513 & $0.052 *$ \\
\hline $\mathrm{C} 9$ & 0.7050 & $0.43722(14)$ & $0.36244(12)$ & $0.0396(4)$ \\
\hline H9A & 0.7276 & 0.5081 & 0.3914 & $0.048^{*}$ \\
\hline Н9B & 0.5827 & 0.4152 & 0.3763 & $0.048 *$ \\
\hline $\mathrm{C} 10$ & 0.8618 & $0.20169(18)$ & $0.12824(14)$ & $0.0495(5)$ \\
\hline C11 & $0.7283(4)$ & $0.25562(19)$ & $0.06728(14)$ & $0.0630(6)$ \\
\hline H11A & 0.6211 & 0.2099 & 0.0617 & $0.076^{*}$ \\
\hline H11B & 0.7805 & 0.2665 & 0.0098 & $0.076^{*}$ \\
\hline $\mathrm{C} 12$ & $0.6842(3)$ & $0.36830(18)$ & 0.10993 (14) & $0.0583(6)$ \\
\hline $\mathrm{H} 12 \mathrm{~A}$ & 0.5613 & 0.3905 & 0.0973 & $0.070^{*}$ \\
\hline H12B & 0.7651 & 0.4264 & 0.0893 & $0.070^{*}$ \\
\hline
\end{tabular}


supporting information

$\begin{array}{lllll}\text { C13 } & 0.7564(3) & 0.05319(14) & 0.47094(13) & 0.0453(5) \\ \text { H13A } & 0.6282 & 0.0623 & 0.4737 & 0.068^{*} \\ \text { H13B } & 0.7992 & 0.0226 & 0.5251 & 0.068^{*} \\ \text { H13C } & 0.7864 & 0.0032 & 0.4240 & 0.068^{*} \\ \text { C14 } & 1.0567(3) & 0.33493(19) & 0.20250(15) & 0.0519(5) \\ \text { H14A } & 1.0488 & 0.3846 & 0.1533 & 0.078^{*} \\ \text { H14B } & 1.1536 & 0.2830 & 0.1937 & 0.078^{*} \\ \text { H14C } & 1.0786 & 0.3777 & 0.2545 & 0.078^{*} \\ \text { C15 } & 0.5664(3) & 0.53701(18) & 0.23775(17) & 0.0650(7) \\ \text { H15A } & 0.5670 & 0.6004 & 0.2764 & 0.098^{*} \\ \text { H15B } & 0.4526 & 0.4992 & 0.2419 & 0.098^{*} \\ \text { H15C } & 0.5847 & 0.5620 & 0.1788 & 0.098^{*} \\ \text { O1 } & 0.79726(18) & 0.22938(12) & 0.60507(9) & 0.0478(3) \\ \text { O2 } & 0.80792(16) & 0.35200(10) & 0.49559(7) & 0.0390(3) \\ \text { O3 } & 0.9465(2) & 0.11791(15) & 0.11224(11) & 0.0721(5)\end{array}$

Atomic displacement parameters $\left(\AA^{2}\right)$

\begin{tabular}{lllllll}
\hline & $U^{11}$ & $U^{22}$ & $U^{33}$ & $U^{12}$ & $U^{13}$ & $U^{23}$ \\
\hline C1 & $0.0265(8)$ & $0.0451(10)$ & $0.0433(11)$ & $-0.0014(8)$ & $-0.0003(8)$ & $0.0008(8)$ \\
C2 & $0.0416(10)$ & $0.0404(9)$ & $0.0400(10)$ & $-0.0014(8)$ & $0.0015(8)$ & $0.0040(8)$ \\
C3 & $0.0265(8)$ & $0.0298(8)$ & $0.0392(9)$ & $-0.0018(7)$ & $0.0004(7)$ & $0.0008(7)$ \\
C4 & $0.0296(9)$ & $0.0335(8)$ & $0.0384(9)$ & $-0.0040(7)$ & $0.0040(7)$ & $-0.0020(7)$ \\
C5 & $0.0314(9)$ & $0.0331(9)$ & $0.0393(10)$ & $0.0025(7)$ & $-0.0013(8)$ & $-0.0011(7)$ \\
C6 & $0.0344(9)$ & $0.0381(9)$ & $0.0387(10)$ & $-0.0011(8)$ & $0.0020(8)$ & $0.0015(8)$ \\
C7 & $0.0381(9)$ & $0.0357(8)$ & $0.0426(10)$ & $0.0007(8)$ & $-0.0058(8)$ & $0.0050(8)$ \\
C8 & $0.0453(11)$ & $0.0301(8)$ & $0.0534(12)$ & $0.0008(9)$ & $0.0005(9)$ & $0.0064(8)$ \\
C9 & $0.0373(10)$ & $0.0303(8)$ & $0.0512(11)$ & $-0.0012(8)$ & $0.0023(9)$ & $-0.0031(7)$ \\
C10 & $0.0526(12)$ & $0.0532(12)$ & $0.0426(12)$ & $0.0015(10)$ & $-0.0019(9)$ & $-0.0027(9)$ \\
C11 & $0.0807(17)$ & $0.0660(14)$ & $0.0424(12)$ & $0.0121(14)$ & $-0.0134(12)$ & $-0.0037(10)$ \\
C12 & $0.0731(15)$ & $0.0544(12)$ & $0.0472(12)$ & $0.0092(12)$ & $-0.0115(12)$ & $0.0079(9)$ \\
C13 & $0.0473(11)$ & $0.0383(10)$ & $0.0502(12)$ & $0.0076(9)$ & $0.0064(9)$ & $0.0056(8)$ \\
C14 & $0.0441(11)$ & $0.0595(12)$ & $0.0522(13)$ & $-0.0096(10)$ & $0.0101(10)$ & $0.0073(10)$ \\
C15 & $0.0772(17)$ & $0.0419(11)$ & $0.0761(17)$ & $0.0178(12)$ & $-0.0123(14)$ & $0.0068(11)$ \\
O1 & $0.0432(8)$ & $0.0636(8)$ & $0.0367(8)$ & $-0.0003(7)$ & $0.0028(6)$ & $0.0023(6)$ \\
O2 & $0.0380(7)$ & $0.0401(7)$ & $0.0388(7)$ & $-0.0038(6)$ & $0.0016(6)$ & $-0.0055(5)$ \\
O3 & $0.0840(12)$ & $0.0763(11)$ & $0.0560(10)$ & $0.0307(10)$ & $-0.0134(9)$ & $-0.0248(9)$ \\
& & & & & &
\end{tabular}

Geometric parameters $\left(A,{ }^{\circ}\right)$

\begin{tabular}{llll}
\hline $\mathrm{C} 1-\mathrm{O} 1$ & $1.203(2)$ & $\mathrm{C} 8-\mathrm{C} 15$ & $1.532(3)$ \\
$\mathrm{C} 1-\mathrm{O} 2$ & $1.349(2)$ & $\mathrm{C} 8-\mathrm{C} 9$ & $1.535(3)$ \\
$\mathrm{C} 1-\mathrm{C} 2$ & $1.506(3)$ & $\mathrm{C} 8-\mathrm{H} 8$ & 0.9800 \\
$\mathrm{C} 2-\mathrm{C} 13$ & $1.485(2)$ & $\mathrm{C} 9-\mathrm{H} 9 \mathrm{~A}$ & 0.9700 \\
$\mathrm{C} 2-\mathrm{C} 3$ & $1.527(2)$ & $\mathrm{C} 9-\mathrm{H} 9 \mathrm{~B}$ & 0.9700 \\
$\mathrm{C} 2-\mathrm{H} 2$ & 0.9800 & $\mathrm{C} 10-\mathrm{O} 3$ & $1.206(2)$ \\
$\mathrm{C} 3-\mathrm{C} 5$ & $1.520(2)$ & $\mathrm{C} 10-\mathrm{C} 11$ & $1.507(3)$ \\
$\mathrm{C} 3-\mathrm{C} 4$ & $1.530(2)$ & $\mathrm{C} 11-\mathrm{C} 12$ & $1.530(3)$
\end{tabular}




\begin{tabular}{|c|c|c|c|}
\hline $\mathrm{C} 3-\mathrm{H} 3$ & 0.9800 & $\mathrm{C} 11-\mathrm{H} 11 \mathrm{~A}$ & 0.9700 \\
\hline $\mathrm{C} 4-\mathrm{O} 2$ & $1.473(2)$ & C11-H11B & 0.9700 \\
\hline $\mathrm{C} 4-\mathrm{C} 9$ & $1.503(2)$ & $\mathrm{C} 12-\mathrm{H} 12 \mathrm{~A}$ & 0.9700 \\
\hline $\mathrm{C} 4-\mathrm{H} 4$ & 0.9800 & $\mathrm{C} 12-\mathrm{H} 12 \mathrm{~B}$ & 0.9700 \\
\hline $\mathrm{C} 5-\mathrm{C} 6$ & $1.545(3)$ & $\mathrm{C} 13-\mathrm{H} 13 \mathrm{~A}$ & 0.9600 \\
\hline $\mathrm{C} 5-\mathrm{H} 5 \mathrm{~A}$ & 0.9700 & C13-H13B & 0.9600 \\
\hline C5-H5B & 0.9700 & $\mathrm{C} 13-\mathrm{H} 13 \mathrm{C}$ & 0.9600 \\
\hline $\mathrm{C} 6-\mathrm{C} 10$ & $1.525(3)$ & $\mathrm{C} 14-\mathrm{H} 14 \mathrm{~A}$ & 0.9600 \\
\hline $\mathrm{C} 6-\mathrm{C} 14$ & $1.546(3)$ & $\mathrm{C} 14-\mathrm{H} 14 \mathrm{~B}$ & 0.9600 \\
\hline $\mathrm{C} 6-\mathrm{C} 7$ & $1.558(3)$ & $\mathrm{C} 14-\mathrm{H} 14 \mathrm{C}$ & 0.9600 \\
\hline $\mathrm{C} 7-\mathrm{C} 8$ & $1.544(3)$ & $\mathrm{C} 15-\mathrm{H} 15 \mathrm{~A}$ & 0.9600 \\
\hline $\mathrm{C} 7-\mathrm{C} 12$ & $1.545(3)$ & C15-H15B & 0.9600 \\
\hline $\mathrm{C} 7-\mathrm{H} 7$ & 0.9800 & $\mathrm{C} 15-\mathrm{H} 15 \mathrm{C}$ & 0.9600 \\
\hline $\mathrm{O} 1-\mathrm{C} 1-\mathrm{O} 2$ & $121.39(17)$ & $\mathrm{C} 9-\mathrm{C} 8-\mathrm{H} 8$ & 107.9 \\
\hline $\mathrm{O} 1-\mathrm{C} 1-\mathrm{C} 2$ & $128.53(18)$ & $\mathrm{C} 7-\mathrm{C} 8-\mathrm{H} 8$ & 107.9 \\
\hline $\mathrm{O} 2-\mathrm{C} 1-\mathrm{C} 2$ & $110.08(15)$ & $\mathrm{C} 4-\mathrm{C} 9-\mathrm{C} 8$ & $116.18(15)$ \\
\hline $\mathrm{C} 13-\mathrm{C} 2-\mathrm{C} 1$ & $114.00(15)$ & $\mathrm{C} 4-\mathrm{C} 9-\mathrm{H} 9 \mathrm{~A}$ & 108.2 \\
\hline $\mathrm{C} 13-\mathrm{C} 2-\mathrm{C} 3$ & $118.92(16)$ & $\mathrm{C} 8-\mathrm{C} 9-\mathrm{H} 9 \mathrm{~A}$ & 108.2 \\
\hline $\mathrm{C} 1-\mathrm{C} 2-\mathrm{C} 3$ & $103.43(14)$ & $\mathrm{C} 4-\mathrm{C} 9-\mathrm{H} 9 \mathrm{~B}$ & 108.2 \\
\hline $\mathrm{C} 13-\mathrm{C} 2-\mathrm{H} 2$ & 106.6 & $\mathrm{C} 8-\mathrm{C} 9-\mathrm{H} 9 \mathrm{~B}$ & 108.2 \\
\hline $\mathrm{C} 1-\mathrm{C} 2-\mathrm{H} 2$ & 106.6 & $\mathrm{H} 9 \mathrm{~A}-\mathrm{C} 9-\mathrm{H} 9 \mathrm{~B}$ & 107.4 \\
\hline $\mathrm{C} 3-\mathrm{C} 2-\mathrm{H} 2$ & 106.6 & $\mathrm{O} 3-\mathrm{C} 10-\mathrm{C} 11$ & $124.8(2)$ \\
\hline $\mathrm{C} 5-\mathrm{C} 3-\mathrm{C} 2$ & $113.67(14)$ & $\mathrm{O} 3-\mathrm{C} 10-\mathrm{C} 6$ & $124.83(19)$ \\
\hline $\mathrm{C} 5-\mathrm{C} 3-\mathrm{C} 4$ & $115.01(14)$ & $\mathrm{C} 11-\mathrm{C} 10-\mathrm{C} 6$ & $110.31(17)$ \\
\hline $\mathrm{C} 2-\mathrm{C} 3-\mathrm{C} 4$ & $102.93(13)$ & $\mathrm{C} 10-\mathrm{C} 11-\mathrm{C} 12$ & $104.58(18)$ \\
\hline $\mathrm{C} 5-\mathrm{C} 3-\mathrm{H} 3$ & 108.3 & $\mathrm{C} 10-\mathrm{C} 11-\mathrm{H} 11 \mathrm{~A}$ & 110.8 \\
\hline $\mathrm{C} 2-\mathrm{C} 3-\mathrm{H} 3$ & 108.3 & $\mathrm{C} 12-\mathrm{C} 11-\mathrm{H} 11 \mathrm{~A}$ & 110.8 \\
\hline $\mathrm{C} 4-\mathrm{C} 3-\mathrm{H} 3$ & 108.3 & $\mathrm{C} 10-\mathrm{C} 11-\mathrm{H} 11 \mathrm{~B}$ & 110.8 \\
\hline $\mathrm{O} 2-\mathrm{C} 4-\mathrm{C} 9$ & $107.71(13)$ & $\mathrm{C} 12-\mathrm{C} 11-\mathrm{H} 11 \mathrm{~B}$ & 110.8 \\
\hline $\mathrm{O} 2-\mathrm{C} 4-\mathrm{C} 3$ & $104.38(13)$ & $\mathrm{H} 11 \mathrm{~A}-\mathrm{C} 11-\mathrm{H} 11 \mathrm{~B}$ & 108.9 \\
\hline $\mathrm{C} 9-\mathrm{C} 4-\mathrm{C} 3$ & $115.32(15)$ & $\mathrm{C} 11-\mathrm{C} 12-\mathrm{C} 7$ & $104.55(16)$ \\
\hline $\mathrm{O} 2-\mathrm{C} 4-\mathrm{H} 4$ & 109.7 & $\mathrm{C} 11-\mathrm{C} 12-\mathrm{H} 12 \mathrm{~A}$ & 110.8 \\
\hline $\mathrm{C} 9-\mathrm{C} 4-\mathrm{H} 4$ & 109.7 & $\mathrm{C} 7-\mathrm{C} 12-\mathrm{H} 12 \mathrm{~A}$ & 110.8 \\
\hline $\mathrm{C} 3-\mathrm{C} 4-\mathrm{H} 4$ & 109.7 & $\mathrm{C} 11-\mathrm{C} 12-\mathrm{H} 12 \mathrm{~B}$ & 110.8 \\
\hline $\mathrm{C} 3-\mathrm{C} 5-\mathrm{C} 6$ & $115.87(13)$ & $\mathrm{C} 7-\mathrm{C} 12-\mathrm{H} 12 \mathrm{~B}$ & 110.8 \\
\hline $\mathrm{C} 3-\mathrm{C} 5-\mathrm{H} 5 \mathrm{~A}$ & 108.3 & $\mathrm{H} 12 \mathrm{~A}-\mathrm{C} 12-\mathrm{H} 12 \mathrm{~B}$ & 108.9 \\
\hline $\mathrm{C} 6-\mathrm{C} 5-\mathrm{H} 5 \mathrm{~A}$ & 108.3 & $\mathrm{C} 2-\mathrm{C} 13-\mathrm{H} 13 \mathrm{~A}$ & 109.5 \\
\hline $\mathrm{C} 3-\mathrm{C} 5-\mathrm{H} 5 \mathrm{~B}$ & 108.3 & $\mathrm{C} 2-\mathrm{C} 13-\mathrm{H} 13 \mathrm{~B}$ & 109.5 \\
\hline $\mathrm{C} 6-\mathrm{C} 5-\mathrm{H} 5 \mathrm{~B}$ & 108.3 & $\mathrm{H} 13 \mathrm{~A}-\mathrm{C} 13-\mathrm{H} 13 \mathrm{~B}$ & 109.5 \\
\hline $\mathrm{H} 5 \mathrm{~A}-\mathrm{C} 5-\mathrm{H} 5 \mathrm{~B}$ & 107.4 & $\mathrm{C} 2-\mathrm{C} 13-\mathrm{H} 13 \mathrm{C}$ & 109.5 \\
\hline $\mathrm{C} 10-\mathrm{C} 6-\mathrm{C} 5$ & $109.98(14)$ & $\mathrm{H} 13 \mathrm{~A}-\mathrm{C} 13-\mathrm{H} 13 \mathrm{C}$ & 109.5 \\
\hline $\mathrm{C} 10-\mathrm{C} 6-\mathrm{C} 14$ & $104.76(16)$ & $\mathrm{H} 13 \mathrm{~B}-\mathrm{C} 13-\mathrm{H} 13 \mathrm{C}$ & 109.5 \\
\hline $\mathrm{C} 5-\mathrm{C} 6-\mathrm{C} 14$ & $109.96(15)$ & $\mathrm{C} 6-\mathrm{C} 14-\mathrm{H} 14 \mathrm{~A}$ & 109.5 \\
\hline $\mathrm{C} 10-\mathrm{C} 6-\mathrm{C} 7$ & $103.01(15)$ & $\mathrm{C} 6-\mathrm{C} 14-\mathrm{H} 14 \mathrm{~B}$ & 109.5 \\
\hline $\mathrm{C} 5-\mathrm{C} 6-\mathrm{C} 7$ & $115.63(14)$ & $\mathrm{H} 14 \mathrm{~A}-\mathrm{C} 14-\mathrm{H} 14 \mathrm{~B}$ & 109.5 \\
\hline $\mathrm{C} 14-\mathrm{C} 6-\mathrm{C} 7$ & $112.70(14)$ & $\mathrm{C} 6-\mathrm{C} 14-\mathrm{H} 14 \mathrm{C}$ & 109.5 \\
\hline $\mathrm{C} 8-\mathrm{C} 7-\mathrm{C} 12$ & $113.76(15)$ & $\mathrm{H} 14 \mathrm{~A}-\mathrm{C} 14-\mathrm{H} 14 \mathrm{C}$ & 109.5 \\
\hline
\end{tabular}


supporting information

$\begin{array}{ll}\mathrm{C} 8-\mathrm{C} 7-\mathrm{C} 6 & 116.85(15) \\ \mathrm{C} 12-\mathrm{C} 7-\mathrm{C} 6 & 103.16(15) \\ \mathrm{C} 8-\mathrm{C} 7-\mathrm{H} 7 & 107.5 \\ \mathrm{C} 12-\mathrm{C} 7-\mathrm{H} 7 & 107.5 \\ \mathrm{C} 6-\mathrm{C} 7-\mathrm{H} 7 & 107.5 \\ \mathrm{C} 15-\mathrm{C} 8-\mathrm{C} 9 & 107.59(17) \\ \mathrm{C} 15-\mathrm{C} 8-\mathrm{C} 7 & 111.13(17) \\ \mathrm{C} 9-\mathrm{C} 8-\mathrm{C} 7 & 114.24(14) \\ \mathrm{C} 15-\mathrm{C} 8-\mathrm{H} 8 & 107.9\end{array}$

$\mathrm{H} 14 \mathrm{~B}-\mathrm{C} 14-\mathrm{H} 14 \mathrm{C}$
C8-C15-H15A
C8-C15-H15B
H15A-C15-H15B
C8-C15-H15C
H15A-C15-H15C
H15B-C15-H15C
C1-O2-C 4

109.5

109.5

109.5

109.5

109.5

109.5

109.5

$110.89(13)$ 